\title{
Wireless Non-contact Biopotential Electrodes
}

\author{
Yu M. Chi, Patrick Ng \\ Department of Electrical and \\ Computer Engineering \\ University of California, San \\ Diego \\ 9500 Gilman Drive \\ La Jolla, CA 92093 \\ m1chi, png@ucsd.edu
}

\author{
Christoph Maier, Gert \\ Cauwenberghs \\ Department of Bioengineering \\ University of California, San \\ Diego \\ 9500 Gilman Drive \\ La Jolla, CA 92093 \\ gert@ucsd.edu
}

\begin{abstract}
Ubiquitous physiological monitoring will be a key driving force in the upcoming wireless health revolution. Cardiac and brain signals in the form of ECG and EEG are two critical health indicators that directly benefit from long-term monitoring. Despite advancements in wireless technology and electronics miniaturization, however, the use of wireless home ECG/EEG monitoring is still limited by the inconvenience and discomfort of wet adhesive electrodes.

We have developed a wireless biopotential instrumentation system using non-contact capacitive electrodes that operate without skin contact. The compact, battery-powered, wireless system accepts inputs from both standard $\mathrm{Ag} / \mathrm{AgCl}$ electrodes and non-contact sensors and provides live telemetry to a laptop computer. We will demonstrate the interactive, prototype ECG/EEG system for acquiring cardiac and brain signals quickly and through clothing.
\end{abstract}

\section{General Terms}

EEG, ECG, Wireless Health, Capacitive Sensors

\section{INTRODUCTION}

Brain and cardiac biopotential signals in the form of EEG and ECG are two critical physiological indicators that are directly suited for long-term wireless health monitoring. Yet despite advancements in wireless technology and electronics miniaturization, however, the use EEG/ECG has still been largely limited by the inconvenience and discomfort of conventional wet contact electrodes.

For home use, clinical grade adhesive electrodes are often cited as irritating and uncomfortable leading to low usage compliance. For future wireless health systems, a less obtrusive sensor is needed to match the advancements made in wireless technology.

In contrast to wet and dry contact sensors, non-contact capacitive electrodes do not require an ohmic connection

Permission to make digital or hard copies of all or part of this work for personal or classroom use is granted without fee provided that copies are not made or distributed for profit or commercial advantage and that copies bear this notice and the full citation on the first page. To copy otherwise, to republish, to post on servers or to redistribute to lists, requires prior specific permission and/or a fee.

Wireless Health 2010, October 5-7, 2010, San Diego, USA

Copyright 2010 ACM 978-1-60558-989-3 ...\$10.00.

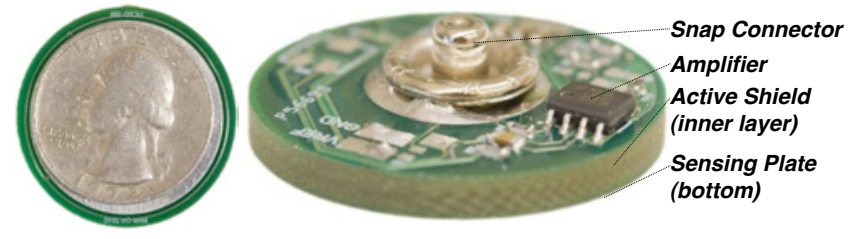

Figure 2: Picture of the non-contact, capacitive electrode. The sensor is manufactured on a standard PCB, which contains the amplifier circuits on the top and the sensing plate on the bottom.

to the body. This offers numerous advantages since noncontact electrodes require zero preparation, are completely insensitive to skin conditions and can be embedded inside a garment for a completely unobtrusive, patient-friendly system.

Our demonstration will show a simple, prototype wireless ECG/EEG system based around these non-contact electrodes.

\section{DEMONSTRATION SYSTEM}

We have developed an ECG chest harness and an EEG headband (Fig. 1) to mount the non-contact electrodes (Fig. 2). Detailed descriptions of the system can be found in the accompanying paper Wireless Non-contact Cardiac and Neural Monitoring.

The prototype system is based around a simple 16-bit micro-controller which controls the data acquisition and telemetry process. The base unit supports both Bluetooth wireless connectivity for live streaming of data to a PC or other display interface. For longer-term mobile recordings, a microSD interface is also available. The entire system is powered from a $900 \mathrm{mAh}$ rechargeable lithium-polymer battery good for approximately 10 hours of continuous recording.

\subsection{Sample Data}

Figure 3 shows a detailed plot of a ECG sample taken while the subject was sitting at rest. The overall signals are nearly indistinguishable in both shape and noise levels, even though the capacitive electrodes were operating through clothing. All relevant ECG features are clearly visible. The slightly smaller amplitude of the signal from the non-contact electrode is likely due to the signal attenuation from the extremely high source impedance.

Similar to the cardiac data, two of the input channels 

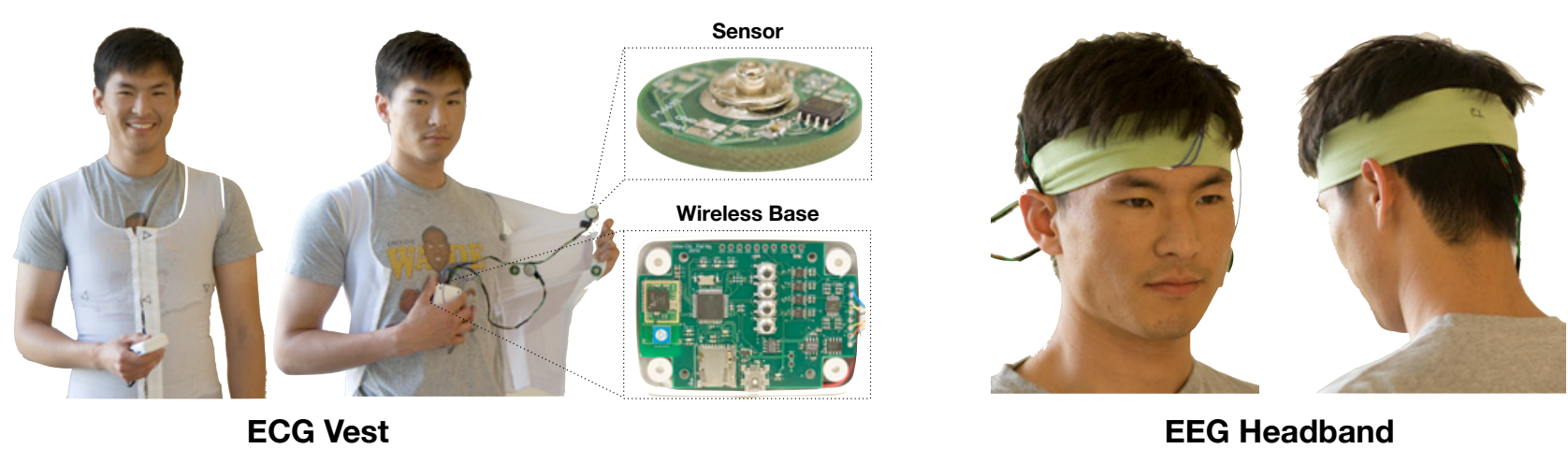

EEG Headband

Figure 1: Picture of the prototype ECG chest vest and EEG head band demonstration system. The demo will showcase the non-contact sensor hardware transmitting telemetry wirelessly to a laptop.

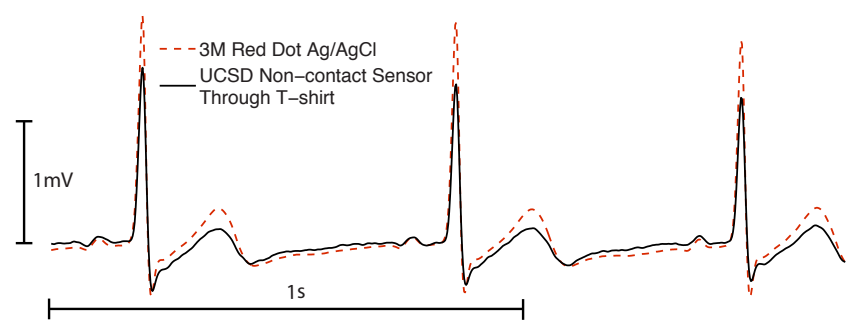

Figure 3: Detailed comparison of signal acquired simultaneously from a set of clinical grade $3 \mathrm{M}$ Red Dot $\mathrm{Ag} / \mathrm{AgCl}$ adhesive electrodes and the noncontact sensor. The non-contact sensor was placed over a cotton t-shirt.

were connected to $\mathrm{Ag} / \mathrm{AgCl}$ adhesive electrodes to achieve a direct comparison with the non-contact sensors. For the EEG experiments, one capacitive electrode was placed on the forehead (Fp1) and the second on the back (Oz), through hair. Likewise, an $\mathrm{Ag} / \mathrm{AgCl}$ electrode was also placed on the forehead (Fp2). However, since it was not possible to place a contact electrode through hair, the second $\mathrm{Ag} / \mathrm{AgCl}$ electrode was placed on the mandible (A1). Unless specified, the output for each electrode was defined as the signal at each channel minus the common-mode of all four channels.

A sample montage consisting of two signals was obtained by using the $\mathrm{A} 1 \mathrm{Ag} / \mathrm{AgCl}$ electrode as a reference for the frontal and parietal capacitive electrodes. In this experiment, the subject was asked to blink several times and then close his eyes. Figure 4 shows the clear blink artifacts in the Fp1A1 signal followed by the onset of alpha waves in the OzA1 signal.

\section{SUMMARY}

We will show a non-contact, wireless ECG/EEG prototype system for acquiring signals easily through clothing. The device is compact and transmits signals to a nearby laptop computer. Audience members will be able to test the system and see live physiological data.

\section{REFERENCES}

[1] R. Matthews, N.J. McDonald, P. Hervieux, P.J. Turner, and M.A. Steindorf. A wearable physiological sensor

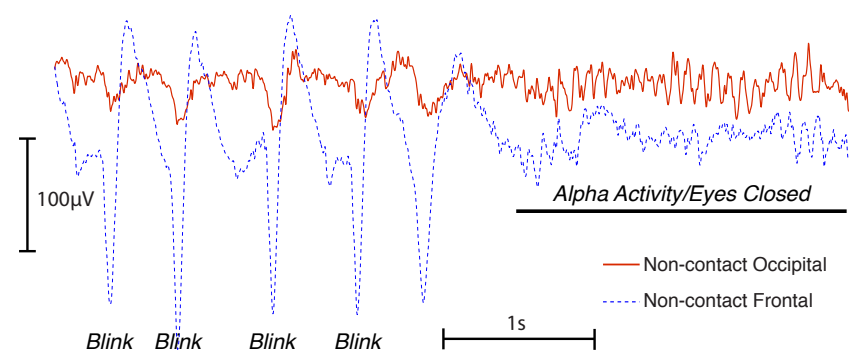

Figure 4: Experiment showing the signal from the frontal capacitive electrode (Fp1A1) in blue and the signal from the occipital capacitive electrode (O1A1). Eye blink artifacts are visible in the frontal electrodes during the first half of the recording. Strong alpha activity is seen in the occipital electrode after the subject's eyes close.

suite for unobtrusive monitoring of physiological and cognitive state. In Engineering in Medicine and Biology Society, 2007. EMBS 200\%. 29th Annual International Conference of the IEEE, pages 5276 -5281, 22-26 2007.

[2] T.J. Sullivan, S.R. Deiss, and G. Cauwenberghs. A low-noise, non-contact eeg/ecg sensor. In Biomedical Circuits and Systems Conference, 200\%. BIOCAS $200 \%$. IEEE, pages 154-157, 27-30 2007.

[3] Yu.M. Chi and G. Cauwenberghs. Micropower non-contact eeg electrode with active common-mode noise suppression and input capacitance cancellation. In Engineering in Medicine and Biology Society, 2009. $E M B C$ 2009. Annual International Conference of the IEEE, pages 4218 -4221, 3-6 2009.

[4] C J Harland, T D Clark, and R J Prance. Electric potential probes - new directions in the remote sensing of the human body. Measurement Science and Technology, 13(2):163, 2002.

[5] M Oehler, V Ling, K Melhorn, and M Schilling. A multichannel portable ecg system with capacitive sensors. Physiological Measurement, 29(7):783, 2008. 